\title{
A functional point-neuron model simulating cochlear nucleus ideal onset responses
}

\author{
Dicke, Ulrike; Dau, Torsten
}

Published in:

Journal of Computational Neuroscience

Publication date:

2005

Document Version

Publisher's PDF, also known as Version of record

Link back to DTU Orbit

Citation (APA):

Dicke, U., \& Dau, T. (2005). A functional point-neuron model simulating cochlear nucleus ideal onset responses. Journal of Computational Neuroscience, 19, 239-253.

\section{General rights}

Copyright and moral rights for the publications made accessible in the public portal are retained by the authors and/or other copyright owners and it is a condition of accessing publications that users recognise and abide by the legal requirements associated with these rights.

- Users may download and print one copy of any publication from the public portal for the purpose of private study or research.

- You may not further distribute the material or use it for any profit-making activity or commercial gain

- You may freely distribute the URL identifying the publication in the public portal

If you believe that this document breaches copyright please contact us providing details, and we will remove access to the work immediately and investigate your claim 
Journal of Computational Neuroscience 19, 239-253, 2005
2005 Springer Science + Business Media, Inc. Manufactured in The Netherlands.

\title{
A Functional Point-Neuron Model Simulating Cochlear Nucleus Ideal Onset Responses
}

\author{
ULRIKE DICKE \\ Medizinische Physik, Carl von Ossietzky Universität Oldenburg, D-26111 Oldenburg, Germany \\ TORSTEN DAU* \\ Centre for Applied Hearing Research, Ørsted•DTU, Technical University of Denmark, \\ DK-2800 Kgs. Lyngby, Denmark \\ tda@oersted.dtu.dk
}

Received June 25, 2004; Revised April 8, 2005; Accepted May 3, 2005

Action Editor: John Rinzel

\begin{abstract}
Cochlear nucleus neurons revealing ideal onset $\left(O_{I}\right)$-type peri-stimulus time histograms (PSTH) encode temporal features of acoustic stimuli with very high precision. These neurons are therefore assumed to be involved in the recognition of natural sounds with temporally varying envelopes such as speech. A functional point-neuron model is presented here for the simulation of $O_{I}$-unit responses found in cochlear nucleus octopus cells. The model assumes a biphasic response of the membrane potential to a current impulse, the membrane impulse response, and a dynamic spike-blocking mechanism. The predicted responses to pure tones at low and high frequencies, injected current steps, and amplitude modulated tones are compared to recordings from the literature. The model accounts for the main response properties in the data using the same small set of parameters for all experimental conditions. The assumed biphasic shape of the membrane impulse response, reflecting a higher sensitivity to stimulus transients and fast changes relative to sustained stimulus portions, allows for a description of $O_{I}$-unit responses that cannot be accounted for by a coincidence detector model with an integrate-to-threshold dynamic. The presented functional model may be useful as a processing module in more complex models of auditory signal processing and perception.
\end{abstract}

Keywords: point-neuron model, ideal onset unit, cochlear nucleus

\section{Introduction}

The cochlear nucleus $(\mathrm{CN})$ is the first nucleus along the ascending auditory pathway in mammals, where the auditory-nerve (AN) fibers synapse on a variety of neurons with different response properties. $\mathrm{CN}$ onset units reveal temporal response properties that differ remarkably from their AN input. While all on-

*To whom correspondence should be addressed. set units exhibit a strong response at stimulus onset, their sustained response differs among the different unit types. Thus, onset units of the ventral cochlear nucleus (VCN) are commonly divided into three groups, based on their peri-stimulus time histograms (PSTH) in response to high-frequency tone bursts: onset with late or long-lasting activity $\left(O_{L}\right)$, onset with chopping $\left(O_{C}\right)$, revealing two or more peaks that are not related to the stimulus frequency, and ideal onset $\left(O_{I}\right)$ (Godfrey et al., 1975; Rhode and Smith, 1986; Winter 
and Palmer, 1995). Despite the ongoing stimulation provided by the AN fibers, $O_{I}$ units show one precisely timed action potential at the tone onset followed by no or little ( $<10$ spikes/s) subsequent activity when stimulated at their characteristic frequency (CF) (e.g., Godfrey et al., 1975; Rhode and Smith, 1986). Another remarkable feature of $O_{I}$ units is their entrainment, i.e., the generation of exactly one spike per stimulus period, in response to low-frequency pure tones, click trains and amplitude modulated (AM) stimuli presented at repetition/modulation rates $<800 \mathrm{~Hz}$. The robust synchronization of $O_{I}$ units in response to AM stimuli is presumably the best among all major cell types in the CN (Frisina et al., 1990; Rhode, 1994; Winter and Palmer, 1995).

$O_{I}$-type PSTHs are associated with morphologically-defined octopus cells (Kane, 1973; Godfrey et al., 1975), that occupy a distinct region in the posteroventral cochlear nucleus (PVCN), called the octopus cell area. The general ability of these cells to entrain to periodic stimuli has led to the assumption that octopus cells may play an important role in the processing of amplitude modulation and pitch information (Golding et al., 1995; Cai et al., 2001). Moreover, since octopus cells emphasize transient stimulus features like gaps and onsets, known to be important in speech processing (e.g., Stevens, 1995) as well as in auditory binding (e.g., Bregman, 1990), it has been suggested that they are also involved in these auditory tasks.

Previous models simulating octopus cell response properties have suggested a number of different mechanisms that might account for octopus cell response properties. Cai et al. (1997, 2000, 2001) implemented a compartmental octopus cell model, that focuses on a detailed description of individual ion conductances, including a low-threshold potassium conductance as well as an inward rectifying mixed cation conductance. Both conductances have been observed in octopus cells (Golding et al., 1999) and are presumed to shape their response properties. The model by Cai et al. (2000) has mainly been tested using injected currents and its entrainment properties to low-frequency tones have not been extensively evaluated. In the one example given (Fig. 9 in Cai et al., 2000) where the model's response to a low-frequency tone was tested, the model did not reliably entrain, failing to generate an action potential every other or few cycles. Another compartmental octopus cell model, suggested by Levy and Kipke (1997) (see also Kipke and Levy, 1997; Levy and Kipke, 1998), includes morphological and electrophys- iological octopus cell properties in order to investigate their role in shaping octopus cell responses. They found two inherent features dominating their model's onset response: dynamic changes in synaptic effectiveness and a dynamically changing spike threshold. However, the simulation results (e.g., Fig. 7 in Levy and Kipke, 1997) reveal that their model also does not produce reliable entrainment to low-frequency pure tones. Thus, while such detailed models allow for investigating the biophysical mechanisms underlying octopus cell responses, using a sufficiently large set of parameters, their ability to actually simulate the distinct response properties for different types of stimuli is limited.

In contrast to compartmental neuron models, the class of point-neuron models provides a more abstract description of biological neurons where spatial variations of the membrane voltage within a cell are neglected. In an integrate-to-threshold neuron model (Tuckwell, 1988), often referred to as the leakyintegrator model, the voltage change due to the flow of synaptic current through the membrane is represented by a parallel resistor-capacitor electrical circuit with a membrane time constant, $\tau_{m}$, equal to $R C$, with $R$ representing the resistance and $C$ the capacitance of the circuit. A spike discharge results when the membrane voltage exceeds the neuron's spike threshold potential. Kalluri and Delgutte (2003a, b) recently presented an integrate-to-threshold point-neuron model in order to investigate possible common properties among neurons with $O_{I}, O_{L}$ and $O_{C}$ types of onset responses. Their model essentially represents a coincidence detector as it is based on a short membrane time constant, $\tau_{m}$, and a large number of weak synaptic AN inputs. Viewing octopus cells as coincidence detectors across their AN input fibers became popular (i) due to the large number of high-spontaneous rate AN fibers (Liberman, 1993) providing across-frequency excitatory AN input and (ii) due to their rapid membrane time constant (<200 $\mu$ s) (Golding et al., 1995, 1999). Moreover, it has been found that an octopus cell is activated only if a sufficient number of AN fibers, causing small and rapid excitatory postsynaptic potentials, are activated within a small time window of approximately $1 \mathrm{~ms}$ (Oertel et al., 2000). Kalluri and Delgutte (2003a) found that their point-neuron coincidence-detector model accounts for $O_{L}$ and $O_{C}$ responses, depending on the specific choice of particular model parameters, while $O_{I}$ unit responses cannot be accounted for. In particular, the entrainment to low frequencies as found in octopus cells cannot be described by using a 
coincidence detector model, as hyper-entrainment (i.e., the generation of more than one spike per stimulus cycle) cannot be avoided.

In order to avoid such hyper-entrainment, Kalluri and Delgutte (2003b) extended their coincidence detector model by a spike-blocking mechanism. Such a mechanism was originally suggested by Romand (1978) as a possible mechanism to account for ideal onset responses. The spike-blocking mechanism implemented by Kalluri and Delgutte is activated after each spike of the neuron, preventing the generation of further spikes. This blocked state lasts until the membrane potential falls below a so-called reactivation threshold and releases the neuron from spike blocking. Kalluri and Delgutte (2003b) showed that their point-neuron coincidence-detector in combination with a spike-blocking mechanism accounts for temporal discharge patterns in response to individual tones in $O_{I}$ units, indicating that some of their responses can be understood from relatively simple principles. However, it will be shown in the present study that, due to its integrate-to-threshold dynamic and its fixed spike-blocking release threshold, the model does not account for $O_{I}$-unit responses to tones covering a wide range of frequencies and sound pressure levels or for octopus cell responses to injected currents (e.g., Oertel et al., 2000; Ferragamo and Oertel, 2002).

The functional point-neuron model described in the present study is conceptually similar to the model by Kalluri and Delgutte (2003b) as it also uses a spikeblocking mechanism (Romand, 1978) with a fixed spike-blocking release threshold. In contrast to a leakyintegrator model (Tuckwell, 1988) that implies an exponentially decaying (monophasic) response to an injected current impulse, the present model assumes a biphasic impulse response. The biphasic shape of the impulse response essentially corresponds to a highpass filtering of the stimulus input, i.e., stimulus transients and fast changes are enhanced, while sustained stimulus portions are attenuated. In response to a sinusoidal stimulus, for example, the simulated membrane potential is driven towards the (fixed) spike-blocking release threshold within each period of the stimulus, even if high stimulus frequencies and high levels are used. Such a "change detector" is different from a coincidence detector (in connection with a spike-blocking mechanism) where the membrane potential follows the stimulus waveform itself. Thus, for high stimulus frequencies and high levels, the integration properties of the coincidence-detector model prevent the membrane potential from falling below the spike-blocking release threshold within each stimulus period. The assumption that $O_{I}$ units act as change detectors on their input is consistent with electrophysiological octopus cell recordings showing that the cells fire only if the rate of rise of a depolarization exceeds a certain threshold value that varies between 5 and $15 \mathrm{mV} / \mathrm{ms}$ among cells (Ferragamo and Oertel, 2002).

As does the model by Kalluri and Delgutte (2003a, b), the present model reflects an abstract model with no direct correspondence to biophysical processes. In contrast to their model, the present model attempts to cover experimental results obtained from $O_{I}$ units only. However, this is done for a larger set of stimuli. The focus of the present study is to simulate the spiking behavior of the neuron in response to the various stimuli, while the underlying biophysical processes are not addressed. In the first part of the paper, the structure of the model and its dynamics are described. This is followed by an analysis of the model's capabilities when tested with pure tones, polarizing current pulses, and amplitudemodulated tones. The simulated results are compared to corresponding experimental data from the literature and to results from the leaky-integration coincidencedetecting model as suggested by Kalluri and Delgutte (2003b).

\section{Methods}

A schematic visualization of the model is shown in Fig. 1. The different components of the model are described in the following.

\subsection{Auditory Periphery}

Basilar-membrane filtering is simulated using a bank of fourth-order gammatone filters (Patterson et al., 1988), with center frequencies spaced according to the equivalent rectangular bandwidth (ERB) scale in humans. The output of each gammatone filter serves as input to Meddis' model of the mechanical to neural transduction at the hair cell-auditory nerve synapse (Meddis, 1986, 1988; Meddis et al., 1990). The output is the instantaneous AN discharge rate function in response to arbitrary stimuli. The discharge rate at a particular point in time can be considered as being proportional to the probability that a spike is generated. In order to account for the decreased phase-locking ability of AN fibers at high frequencies, the AN activity 


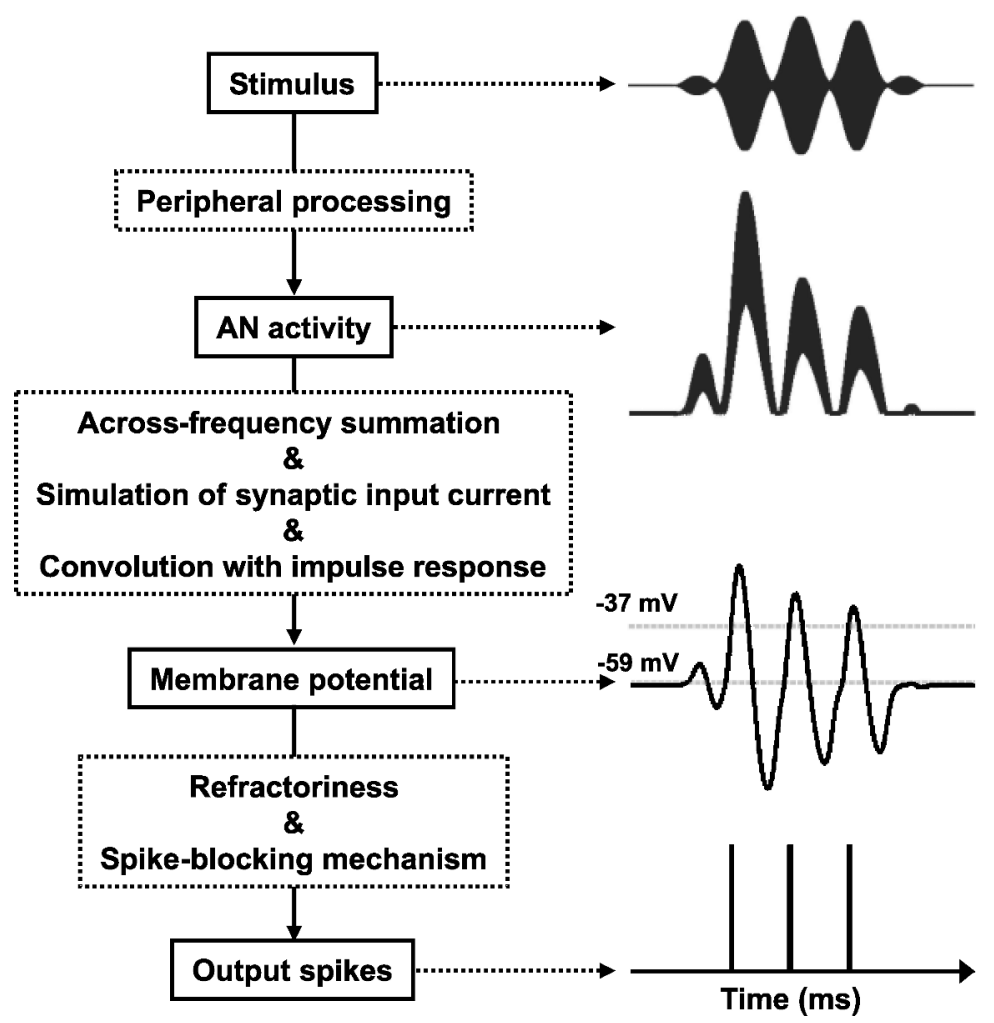

Figure 1. Model scheme and example intermediate representations: An acoustic stimulus (top) provides the input to the peripheral processing model, resulting in the simulated AN activity. This activity, integrated across-frequency, is convolved with a exponentially decaying function yielding the synaptic input current to the model. The model membrane potential results from the convolution of the synaptic input with the biphasic membrane impulse response of the model. If the membrane potential exceeds the spike threshold $\Theta_{\text {act }}=-37 \mathrm{mV}$, a spike is generated. Following a spike, the model enters its refractory period and its spike-blocking mechanism becomes activated. The spike-blocking mechanism remains active until the model membrane potential falls below the model's spike-blocking release threshold $\Theta_{\text {rel }}=-59 \mathrm{mV}$. The model's output spike train in response to the acoustic stimulus is shown at the bottom.

function within each cochlear channel is low-pass filtered using a second-order Butterworth filter with a cutoff frequency of $900 \mathrm{~Hz}$. In the present implementation it is assumed that a large number of AN fibers converge on a $\mathrm{CN}$ model unit. Therefore, the deterministic discharge rate function is used as the timedependent AN input to the model, i.e., no individual spike trains for each AN fiber are computed. Such a convergent input agrees with observations from octopus cell recordings, although the actual number of AN fibers projecting onto an octopus cell has been difficult to estimate (e.g., Liberman, 1993; Oertel et al., 2000). In order to account for the wide across-frequency input that octopus cells receive (e.g., Oertel et al., 2000), the simulated AN activity is summed across a range of cochlear filters, such that each model unit receives equally weighted input from 11 cochlear filters spanning a frequency range of approximately one octave centered at the unit's CF. In order to obtain the model input current from the output of the hair-cell model, it is multiplied by a constant synaptic conductance of $20 \mathrm{nS}$ and convolved with an exponential function that decays with a time constant of $0.35 \mathrm{~ms}$. With this choice of the synaptic conductance, a total synaptic input current of approx. $3 \mathrm{nA}$ is generated in the $4 \mathrm{kHz}$ unit, as an example, if it is stimulated with a $4-\mathrm{kHz}$ pure tone at threshold. The time constant corresponds to the decay time of a miniature excitatory postsynaptic current (mEPSC) observed in octopus cells (e.g., Gardner et al., 1999).

\subsection{Model Dynamics}

The functional octopus cell is modeled as a point neuron. In contrast to compartmental octopus cell models (e.g., Cai et al., 1997, 2000, 2001; Levy and Kipke, 

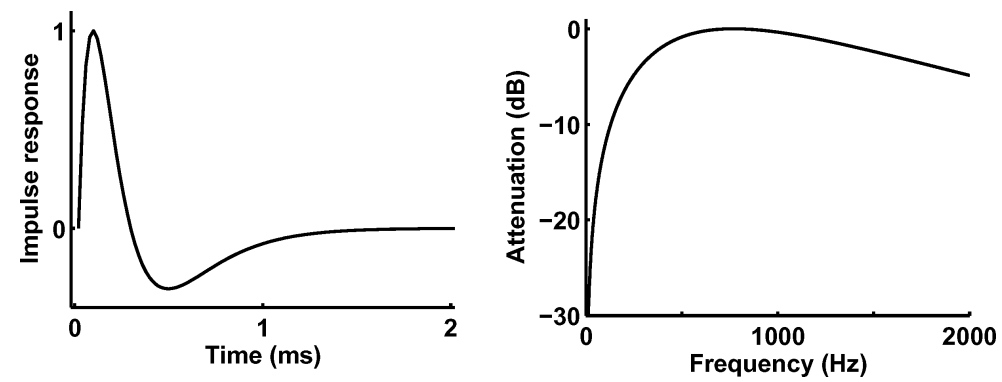

Figure 2. Left panel: Biphasic membrane impulse response assumed in the present model. Right panel: Corresponding magnitude transfer function of the impulse response.

1997, 1998; Kipke and Levy, 1997, 1998), a point neuron does not consider any spatial properties of the neuron, i.e., it assumes the same membrane potential everywhere inside the cell. The membrane potential $V(t)$ of the unit is obtained by convolving the input current $I(t)$ with the impulse response $h(t)$ of the model unit:

$$
V(t)=V_{\text {rest }}+R[h(t) * I(t)]
$$

where $V_{\text {rest }}=-60 \mathrm{mV}$ represents the resting potential of the model unit and $R=2 \mathrm{M} \Omega$ denotes its input resistance. Both parameters were chosen to mimic octopus cell recordings that reveal resting potentials of about $-60 \mathrm{mV}$ (e.g., Golding et al., 1999; Bal and Oertel, 2001) and input resistances that range from 2 to $6 \mathrm{M} \Omega$ (e.g., Bal and Oertel, 2001; Golding et al., 1999).

The assumed impulse response of the model unit has a biphasic shape and is chosen as:

$$
h(t)= \begin{cases}\frac{t}{k}\left[e^{\left(-\frac{t}{\tau_{a}}\right)}-c e^{\left(-\frac{t}{\tau_{b}}\right)}\right] & \text { for } t>0 \\ 0 & \text { otherwise }\end{cases}
$$

with the time constants $\tau_{a}=0.1 \mathrm{~ms}, \tau_{b}=0.2 \mathrm{~ms}, c=$ 0.2494 denoting a constant factor, and $k=0.0226 \mathrm{~ms}$ representing a normalizing factor. The model time constants were chosen close to the rapid membrane time constants observed in octopus cells near rest which are in the range of $0.2 \mathrm{~ms}$ (e.g., Golding et al., 1999). The constant $c$ ensures that the integral of the impulse response is zero. Figure 2 shows the impulse response of the unit (left panel), and the corresponding Fourier transform (right panel). The magnitude transfer function has the form of a broad bandpass filter with a maximum at about $800 \mathrm{~Hz}$. In the relevant frequency range from 0 to about $1 \mathrm{kHz}$, the unit effectively acts as a highpass filter on the synaptic input. Therefore, it does not correspond to a leaky integrator.
As long as the cell's membrane potential, $V(t)$, is below its spike threshold $\Theta_{\text {act }}$, the unit's state variable $s$ is set to zero. If the membrane potential of the model unit exceeds the unit's spike threshold, the neuron emits a spike, i.e., its state variable is set to one:

$$
s= \begin{cases}0: & V(t) \leq \Theta_{\mathrm{act}} \\ 1: & V(t)>\Theta_{\mathrm{act}}\end{cases}
$$

The spike threshold $\Theta_{\text {act }}$ was chosen to be $-37 \mathrm{mV}$ in order to account for results from electrophysiological recordings where octopus cells were found to initiate an action potential in response to an injected step current of $1.5 \mathrm{nA}$ (Ferragamo and Oertel, 2002). After the simulated neuron has emitted a spike, it remains in an absolute refractory period with a duration of $0.7 \mathrm{~ms}$, preventing it from emitting further action potentials $(s=0)$. The value of the absolute refractory period was chosen to correspond to the smallest interspike intervals observed in VCN neurons (e.g., Young et al., 1988). In addition, a spike-blocking mechanism is activated following each spike, which also prevents the unit from emitting further spikes unless its membrane potential falls below the spike-blocking release potential $\Theta_{\text {rel }}=-59 \mathrm{mV}$.

Simulations obtained with the present model will be compared with simulations obtained with the leakyintegrator model as suggested by Kalluri and Delgutte (2003b). The passive electrical properties of a leaky integrator can be effectively described by an equivalent electrical circuit based on the membrane capacitance $(C)$ and the internal resistance $(R)$. The product $R C$ reflects the integration time constant $\tau_{m}$ of the membrane. The impulse response of such a circuit differs from the biphasic impulse response assumed in the present 
model as it decays exponentially:

$$
h(t)= \begin{cases}e^{\left(-\frac{t}{\tau_{m}}\right)} & \text { for } t>0 \\ 0 & \text { otherwise }\end{cases}
$$

The membrane time constant, $\tau_{m}=0.125 \mathrm{~ms}$, was chosen according to Kalluri and Delgutte (2003b). Results from the leaky-integrator model were obtained by using this exponentially decaying impulse response in Eq. (1) instead of the biphasic impulse response. The spike-blocking release threshold was chosen to be $-50.8 \mathrm{mV}$. This corresponds to 0.4 times the potential difference between the unit's resting potential and its spike threshold, a value that was also used by Kalluri and Delgutte (2003b). With the exception of these two parameters, the leaky-integrator model uses the same parameters as the present model.

\section{Results}

The presented $O_{I}$ model was tested using pure tones, polarizing currents, and amplitude-modulated (AM) tones. The simulations are compared to literature data from octopus cell recordings, if available, otherwise they are compared to $O_{I}$ unit data. The model responses to the pure-tone stimuli are analyzed in terms of their peri-stimulus time histograms (PSTH). The degree of synchronization of the model response to AM stimuli is analyzed using the synchronization coefficient (SC) (Goldberg and Brown, 1969), computed as the vector strength of the distribution of spikes. SC $=1$ indicates perfect synchronization to the stimulus modulation while $\mathrm{SC}=0$ represents no synchronization at all. Additionally, the rate modulation transfer function (rMTF), where the spike rate is plotted as a function of the modulation frequency, is computed in response to AM stimuli. Stimulus levels are always given in $\mathrm{dB}$ above the individual CF pure-tone response threshold of a model unit (mTh). This individual threshold depends on whether the present model or the leakyintegrator model is used, as well as on the CF of the simulated unit. The pure-tone response thresholds of all units used in the simulations are within a range of $8 \mathrm{~dB}$. All simulations of the present study were performed at a sampling rate of $50 \mathrm{kHz}$.

\section{1. $\quad$ Pure-Tone Stimuli}

Godfrey et al. (1975) found that $O_{I}$ unit responses to pure-tone stimuli $(f>2 \mathrm{kHz})$ reveal one sharply timed action potential at stimulus onset followed by no or little ( $<10$ spikes/s) subsequent activity during the sustained portion of the tone. He concluded that these ideal onset responses that were confirmed in a number of subsequent studies (e.g., Rhode and Smith, 1986; Rhode, 1994) can be associated with octopus cell responses. In contrast to their onset response at high stimulus frequencies, $O_{I}$ units can entrain to low-frequency pure tones $(f<800 \mathrm{~Hz})$, where they emit exactly one spike at each stimulus cycle (e.g., Rhode and Smith, 1986), if the stimulus frequency is included in the cell's frequency-response area. The synchronization coefficient of low-frequency pure-tone entrainment lies between 0.9 and 0.99 (Rhode and Smith, 1986), indicating a nearly perfect synchronization of the cell's response to the stimulus frequency.

The left panel of Fig. 3 shows, as an example, the PSTH of a model cell $(\mathrm{CF}=4 \mathrm{kHz})$ in response to a $4-$ $\mathrm{kHz}$ pure tone presented at $60 \mathrm{~dB}(\mathrm{mTh})$. Such reliable onset responses are produced by the model for all suprathreshold stimulus levels tested (not shown). Due to the
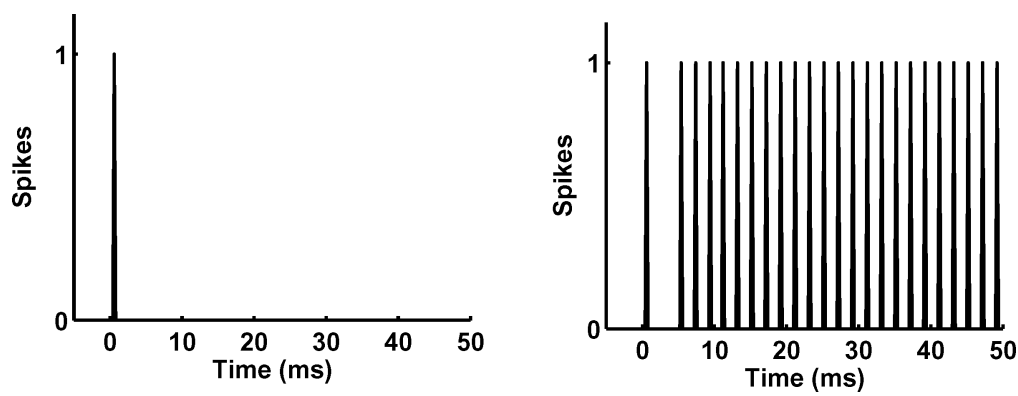

Figure 3. Left panel: PSTH of a model unit $(\mathrm{CF}=4 \mathrm{kHz})$ in response to a 4-kHz tone presented at $60 \mathrm{~dB}(\mathrm{mTh})$. Right panel: PSTH of the same model unit in response to a $500-\mathrm{Hz}$ tone presented at $60 \mathrm{~dB}(\mathrm{mTh})$. Note that the same model unit reveals an onset response when stimulated with high-frequency tones and entrainment when stimulated with low-frequency tones. 

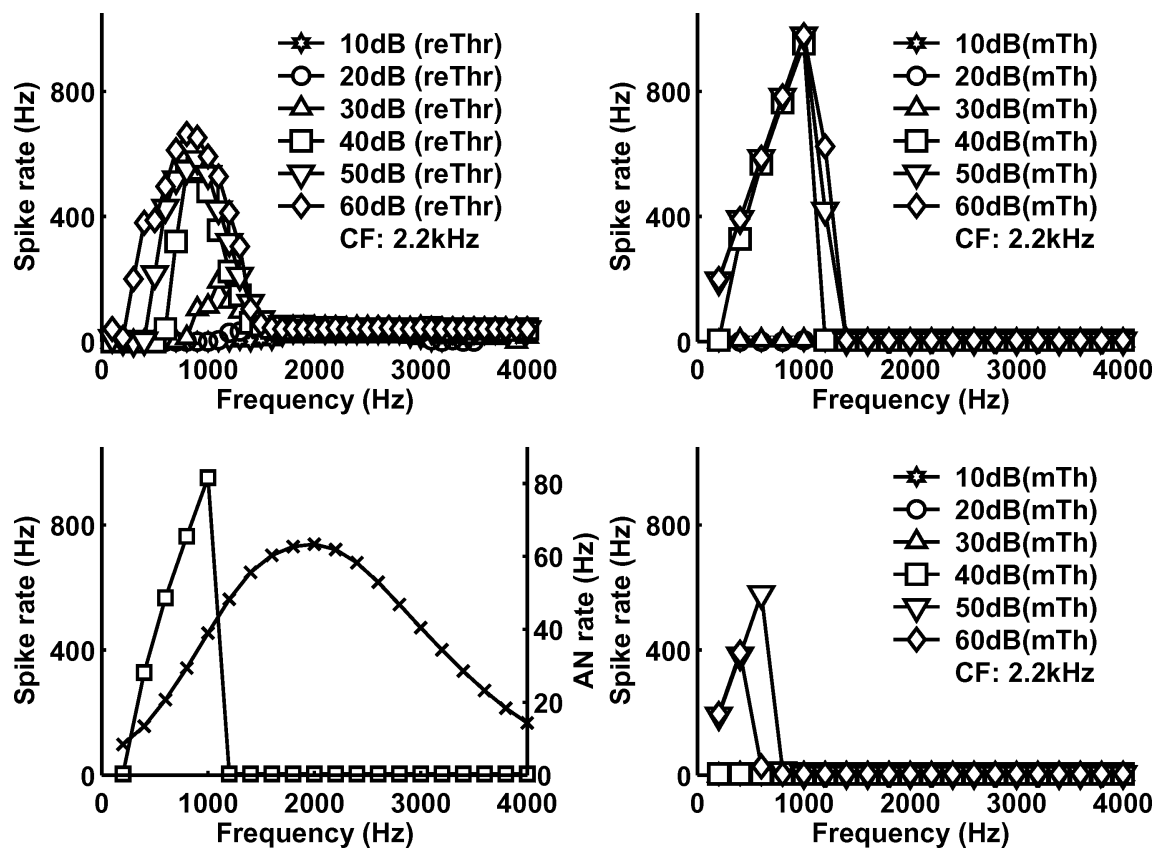

Figure 4. Upper left panel: Recorded $O_{I}$-unit response area $(\mathrm{CF}=2.2 \mathrm{kHz}$, response threshold at $\mathrm{CF}=30 \mathrm{~dB}$ SPL, stimulus duration $250 \mathrm{~ms}$, 250 stimulus repetitions) for stimulus presentation levels from 10 to $60 \mathrm{~dB}$ above the unit's pure-tone threshold (reThr). Replot with permission from Rhode and Smith (1986). Upper right panel: Simulated frequency-response areas of a model unit with $\mathrm{CF}=2.2 \mathrm{kHz}$ for stimulus presentation levels from 10 to $60 \mathrm{~dB}(\mathrm{mTh})$ (stimulus duration $250 \mathrm{~ms}$ ). Lower left panel: Simulated frequency-response area of the present model for a stimulus level of $40 \mathrm{~dB}(\mathrm{mTh})$ (left scale, squares), shown together with the across-frequency AN input (crosses) divided by the number of cochlear input channels the unit receives. Lower right panel: Corresponding frequency-response areas obtained with the leaky-integrator model.

spike-blocking mechanism, multiple spikes do not occur at stimulus onset, not even at the highest stimulation level tested $(90 \mathrm{~dB}(\mathrm{mTh}))$. If the same model unit is stimulated with a low-frequency pure tone, it entrains to the stimulus frequency. This can be seen in the right panel of Fig. 3, where the simulated PSTH of the model unit $(\mathrm{CF}=4 \mathrm{kHz})$ is shown in response to a $500-\mathrm{Hz}$ tone presented at $60 \mathrm{~dB}(\mathrm{mTh})$. Although the model fails to generate one spike following the onset of the tone, it reliably entrains to the $500-\mathrm{Hz}$ tone throughout the remaining stimulus. The synchronization coefficient, calculated from the model PSTH, is 0.99. This is in good agreement with recorded synchronization coefficients of $O_{I}$ units (Rhode and Smith, 1986).

The frequency-response area of a neuron reflects the spike rate of the neuron in response to pure tones as a function of frequency, with pure-tone level as the parameter. The upper left panel of Fig. 4 shows a recorded frequency-response area of an $O_{I}$ unit $(\mathrm{CF}=2.2 \mathrm{kHz})$, replotted from Rhode and Smith (1986). The CF puretone threshold was at $30 \mathrm{~dB}$ SPL, and the stimulus level was increased in 10-dB steps from 10 to $60 \mathrm{~dB}$ with respect to the unit's pure-tone threshold (reThr). The frequency-response area is broad and reveals an asymmetric shape with respect to the unit's CF. Such an asymmetric frequency-response area is a particular feature of $O_{I}$ units that receive input from low-frequency cochlear channels. The dynamic range of the recorded $O_{I}$ unit is small when the stimulus frequency equals the unit's CF $(2.2 \mathrm{kHz})$. The only major change in spike rate occurs between 0 and $10 \mathrm{~dB}$ (reThr) where the unit starts do exhibit its onset response while essentially no changes occur when the stimulus level is changed from 10 to $60 \mathrm{~dB}$ (reThr). For lower stimulus frequencies, where entrainment occurs, the observed dynamic range is enlarged. The upper right panel of Fig. 4 shows the simulated frequency-response area of a model neuron with $\mathrm{CF}=2.2 \mathrm{kHz}$ in response to pure-tone stimuli with levels from 10 to $60 \mathrm{~dB}$ (mTh), increased in $10 \mathrm{~dB}$ steps. The simulated frequencyresponse area shows some of the characteristics of the recorded data. The asymmetric shape with respect to $\mathrm{CF}$ can be explained considering the pure-tone PSTHs shown in Fig. 3. While the simulated cell reveals purely 
an onset response at high stimulus frequencies (left panel of Fig. 3) it entrains to low-frequency pure tones (right panel of Fig. 3) with a spike rate that reflects the stimulus frequency. Thus, the cell's entrainment leads to an enhanced spike rate in response to low-frequency tones compared to the spike rate that results from its onset response to high-frequency tones; this causes the asymmetry in the response area. The lower left panel of Fig. 4 displays the model results for a stimulus level of $40 \mathrm{~dB}$ (mTh) (left scale, squares), as a replot from one of the curves in the upper right panel, together with the across-frequency AN input that is received by the unit (right scale, crosses). The figure illustrates the transformation performed by the unit: although it receives symmetrical $\mathrm{AN}$ input with respect to its $\mathrm{CF}$, the unit has an asymmetric frequency-response area due to its entrainment to low frequencies. However, the peak spike rate of the model clearly exceeds the peak spike rate observed in the data, indicating that the model in its present form is too sensitive to stimulus changes when compared to the recorded data. While the dynamic range of the simulated $\mathrm{AN}$ input at $\mathrm{CF}(2.2 \mathrm{kHz})$ exceeds $30 \mathrm{~dB}$ (not shown) there is virtually no dynamic range in the simulated model response. This is partly due to the all-or-nothing behavior of the deterministic model and contrasts the recorded data even if the recorded dynamic range for frequencies at $\mathrm{CF}$ is typically small (about $10 \mathrm{~dB}$ ).

The corresponding simulated frequency-response area obtained with the leaky-integrator model in combination with a spike-blocking mechanism (Kalluri and Delgutte, 2003b) is shown in the lower right panel of Fig. 4. While the leaky-integrator model reveals a similar behavior as the present model when tested for the specific pure tones considered in Fig. 3, its frequency-response area displays a fundamental limitation of the model. At a given stimulus level, only a small range of frequencies exists for which the fluctuations in the membrane potential (that corresponds to a low-pass filtered version of the stimulus) extend the potential difference between the spike threshold and the spike-blocking release threshold. This can be seen, for example, from the model's response to puretones presented at $60 \mathrm{~dB}(\mathrm{mTh})$, represented by the diamonds, where the model exclusively entrains to frequencies of 200 and $400 \mathrm{~Hz}$. Moreover, in contrast to the recorded frequency-response area, the highest frequency to which the model entrains decreases with increasing stimulus level. For example, at a stimulus level of $50 \mathrm{~dB}(\mathrm{mTh})$, represented by the downward pointing triangles, the model unit entrains to pure tones with frequencies between 200 and $600 \mathrm{~Hz}$. Changing the spikeblocking release threshold would not solve this problem, since there always would remain a trade-off between the stimulus level and the highest frequency for which entrainment occurs within the leaky-integrator model. As for the change-detection model, the leakyintegration (coincidence-detecting) model shows virtually no dynamic range, in contrast to the recorded data. This is the case since the two models in their current implementation are deterministic and thus reflect an "all-or-nothing" behavior.

\subsection{Current Stimulation}

The assumption that octopus cells act as change detectors is supported by recordings from Ferragamo and Oertel (2002). They found that, while a square current of $1.5 \mathrm{nA}$ was sufficient to bring an octopus cell to threshold, more than $3 \mathrm{nA}$ were needed to bring the same cell to threshold when the current rose over a $1.2 \mathrm{~ms}$ ramp. Responses of the model unit to current injections of different strengths and rise times are shown in the left panel of Fig. 5. The figure shows the membrane potential (middle) including the unit's spike threshold (dashed line) and the corresponding output spikes (top) in response to three different injected currents (bottom): a square current of $1.5 \mathrm{nA}$ (dotted), and two currents of $2.5 \mathrm{nA}$ (dash-dotted) and $3.2 \mathrm{nA}$ (solid) with a rise time of $1.2 \mathrm{~ms}$. The figure indicates that, while the square current of $1.5 \mathrm{nA}$ is sufficient to bring the unit to threshold, such that it exhibits a spike, the rising current of $2.5 \mathrm{nA}$ does not bring the unit to threshold. Moreover, in agreement with the recordings by Ferragamo and Oertel (2002), a current of $3.2 \mathrm{nA}$ is needed to bring the unit to threshold if the current rises over a $1.2 \mathrm{~ms}$ ramp. Thus, the present model simulates the recorded octopus cell spiking behavior by analyzing the rate of change of the injected current. The right panel of Fig. 5 displays the corresponding responses of the leaky-integrator model for comparison. As can be seen from the figure, the rise time of the injected current has essentially no effect on whether the leaky integrator reaches threshold or not. Only the total strength of the current determines whether the unit generates a spike. While the present model detects the rate of change in the injected current, the leaky-integrator model cannot reproduce this feature observed in octopus cell recordings. 

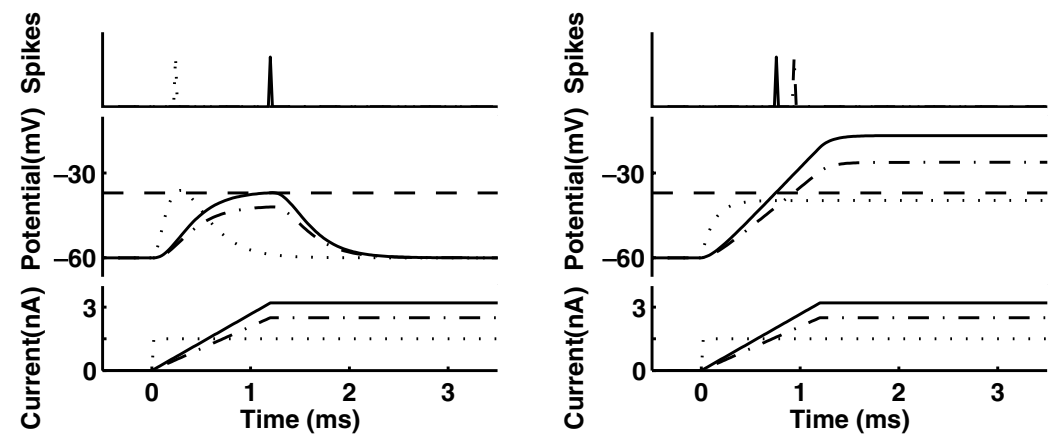

Figure 5. Left panel: Simulated spikes (top) and membrane potential (middle) of the present model in response to injected currents of different strengths and rise times (bottom). Dotted line: square current of $1.5 \mathrm{nA}$, dash-dotted line: current of $2.5 \mathrm{nA}$ with a rise time of $1.2 \mathrm{~ms}$, solid line: current of $3.2 \mathrm{nA}$ with a rise time of $1.2 \mathrm{~ms}$. Right panel: Corresponding results from the leaky-integrator model.
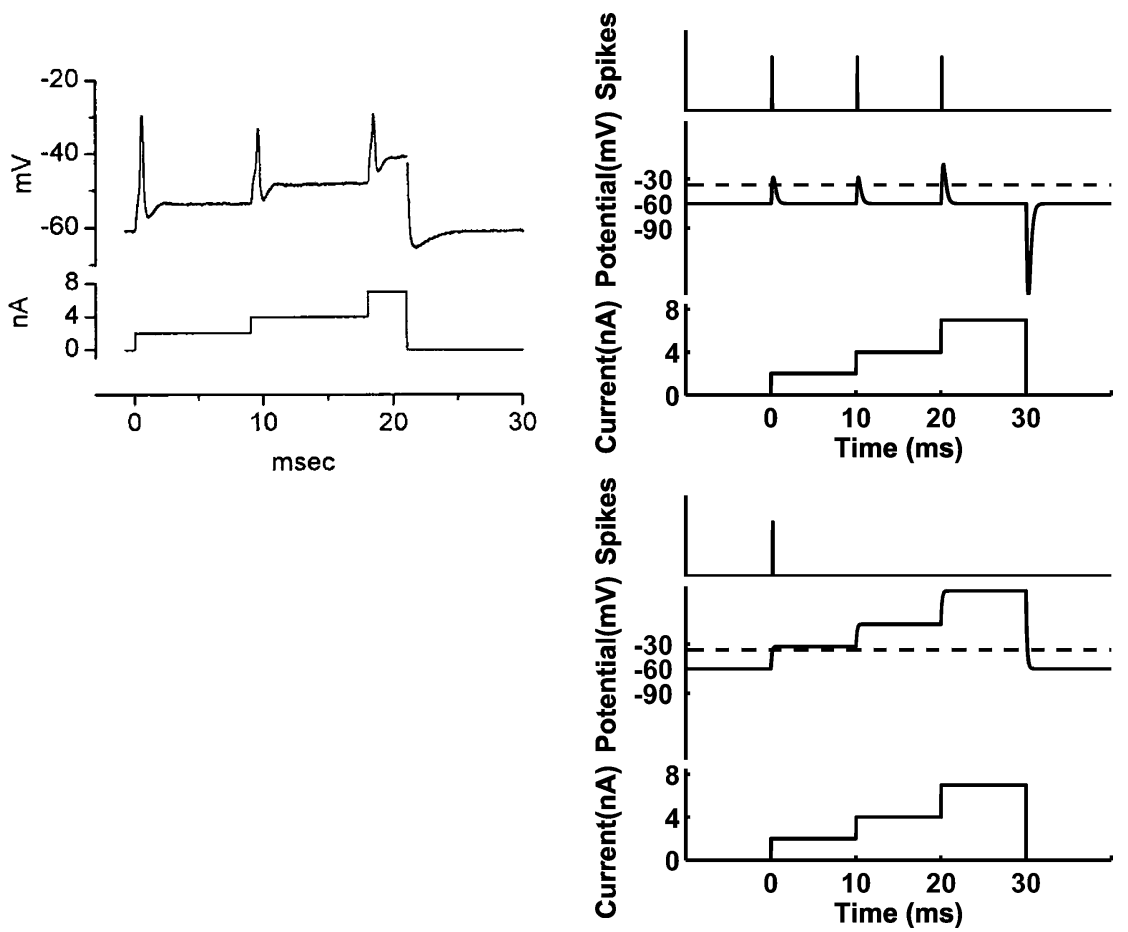

Figure 6. Left panel: Recorded octopus cell data, taken from Oertel et al. (2000) with permission (Copyright (2000) National Academy of Sciences, U.S.A.). The octopus cell potential is shown above the staircase current that was increased from 2 to $4 \mathrm{nA}$ and from 4 to $7 \mathrm{nA}$. Upper right panel: Simulated spike train (top) and the corresponding model membrane potential (middle) of the present model. The vertical dashed line indicates the spike threshold of the model unit. The simulated staircase current (bottom) increased from 2 to $4 \mathrm{nA}$ and from 4 to $7 \mathrm{nA}$. Lower right panel: Corresponding results obtained with the leaky-integrator model.

The left panel of Fig. 6 shows recorded octopus cell data in response to increasing current steps, reprinted from Oertel et al. (2000). When the cell is depolarized with a current pulse of $2 \mathrm{nA}$, it generates an action potential at the onset of the current pulse and remains at a low depolarization during the sustained portion of the current. Further increments of the depolarizing current pulse from 2 to $4 \mathrm{nA}$ and from 4 to $7 \mathrm{nA}$ cause the octopus cell to exhibit further action potentials. After stimulus offset, a hyperpolarization of the octopus cell is observed. The upper right panel of Fig. 6 shows the simulated membrane potential (middle) and 
the corresponding output spike train (top) in response to a staircase current (bottom). The simulated membrane potential exceeds its spike threshold at the beginning of each step, such that the unit emits a spike. The model describes the three spikes as well as the hyperpolarization at the stimulus offset, in qualitative agreement with the data. However, the hyperpolarization of the model membrane potential clearly exceeds that observed in recorded octopus cell responses. Moreover, the simulated membrane potential approaches its resting potential during the sustained stimulus portions and therefore does not show an increased response to an increased current strength in the plateau regions. The reason for this behavior is that the model does not include stimulus integration (see also discussion). The lower right panel of Fig. 6 displays the response of the leaky-integrator model to the same stimulus, for direct comparison. The model exhibits only one spike at stimulus onset and no further spikes since the membrane potential does not fall below the spike-blocking release threshold during the sustained stimulus portions. This behavior is independent of the exact choice of the model's spike-blocking release threshold. In addition, the hyperpolarization observed in octopus cells after stimulus offset is not predicted by the leaky-integrator model because its membrane potential corresponds to a low-pass filtered version of the input current.

Octopus cell recordings using hyperpolarizing current stimuli (Oertel et al., 2000, not shown here) further suggest that a change-detection mechanism might be more appropriate to describe octopus cell spiking behavior than a leaky-integration model. When stimulated with hyperpolarizing currents of sufficient magnitude, octopus cells generate an offset action potential after the hyperpolarizing current has terminated. Such an offset action potential is also generated by the present model when stimulated with hyperpolarizing current steps exceeding $-1.5 \mathrm{nA}$ (not shown). In contrast, the leaky-integrator model with its monophasic impulse response cannot exhibit an offset action potential in response to hyperpolarizing current steps. Thus, in summary, even though the membrane potential is not described correctly by the present model, the spiking behavior can be accounted for when tested with different types of injected currents.

\subsection{Amplitude Modulated Stimuli}

$O_{I}$ units are presumably the best AM encoders among all major CN unit types (Frisina et al., 1990; Rhode,
1994; Winter and Palmer, 1995). Although physiological AM data from isolated octopus cells are limited, responses of $O_{I}$ units to $200 \%$ amplitude modulated tones have been investigated (Rhode, 1994). The left panel of Fig. 7 shows data from Rhode (1994). It represents the recorded synchronization coefficient (SC) (left scale, open triangles) and the rate modulation transfer function (rMTF) (right scale, filled triangles) of an $O_{I}$ unit with $\mathrm{CF}=7 \mathrm{kHz}$ (pure-tone threshold at CF is $0 \mathrm{~dB}$ SPL) in response to a $200 \%$ amplitude modulated $7-\mathrm{kHz}$ tone. The stimulus level was $30 \mathrm{~dB}$ SPL and the stimulus duration was $100 \mathrm{~ms}$. The unit exhibits high envelope synchrony, independent of the stimulus modulation frequency, and a rMTF exhibiting a bandpass shape with a maximum at $450 \mathrm{~Hz}$. The upper right panel of Fig. 7 shows the SC (left scale, open triangles) and the rMTF (right scale, filled triangles) of a model unit $(\mathrm{CF}=7 \mathrm{kHz})$ in response to the same $200 \%$ amplitude modulated $7-\mathrm{kHz}$ tones presented at $30 \mathrm{~dB}(\mathrm{mTh})$. In order to allow for a direct comparison with the recorded data, the analysis window included the whole $100 \mathrm{~ms}$ of the unit's response. The model shows entrainment to the stimulus modulation revealing a high synchronization, and predicts a rMTF that also exhibits a bandpass shape with a maximum at $450 \mathrm{~Hz}$. This best modulation frequency (BMF) results from the interplay of the AN low-pass filter, the membrane time constants, and the assumed thresholds in the model. However, the spike rate of the model, directly resulting from its entrainment property, is larger than that obtained in the recorded rMTF. In addition, the spike rate at frequencies above $450 \mathrm{~Hz}$ where the unit exhibits an onset response instead of entrainment drops rapidly due to the deterministic nature of the model while it decreases gradually in the recordings.

The corresponding results obtained with the leakyintegrator model are shown in the lower right panel of Fig. 7. At a modulation frequency of $50 \mathrm{~Hz}$, the model does not only entrain to the center peak but also to the small lobe of the $200 \%$ AM tone. This results in a doubled spike rate $(100 \mathrm{~Hz})$ and thus in a reduced SC. For modulation frequencies above $50 \mathrm{~Hz}$, the leakyintegrator model does not detect the fluctuations in the stimulus envelope and exhibits an onset response instead of entrainment to the envelope. Although the individual CF pure-tone response thresholds of the leakyintegrator model unit and the present model unit, tested with $200 \%$ amplitude modulated pure tones, differ by only $3 \mathrm{~dB}$, the leaky integrator detects the fluctuations 

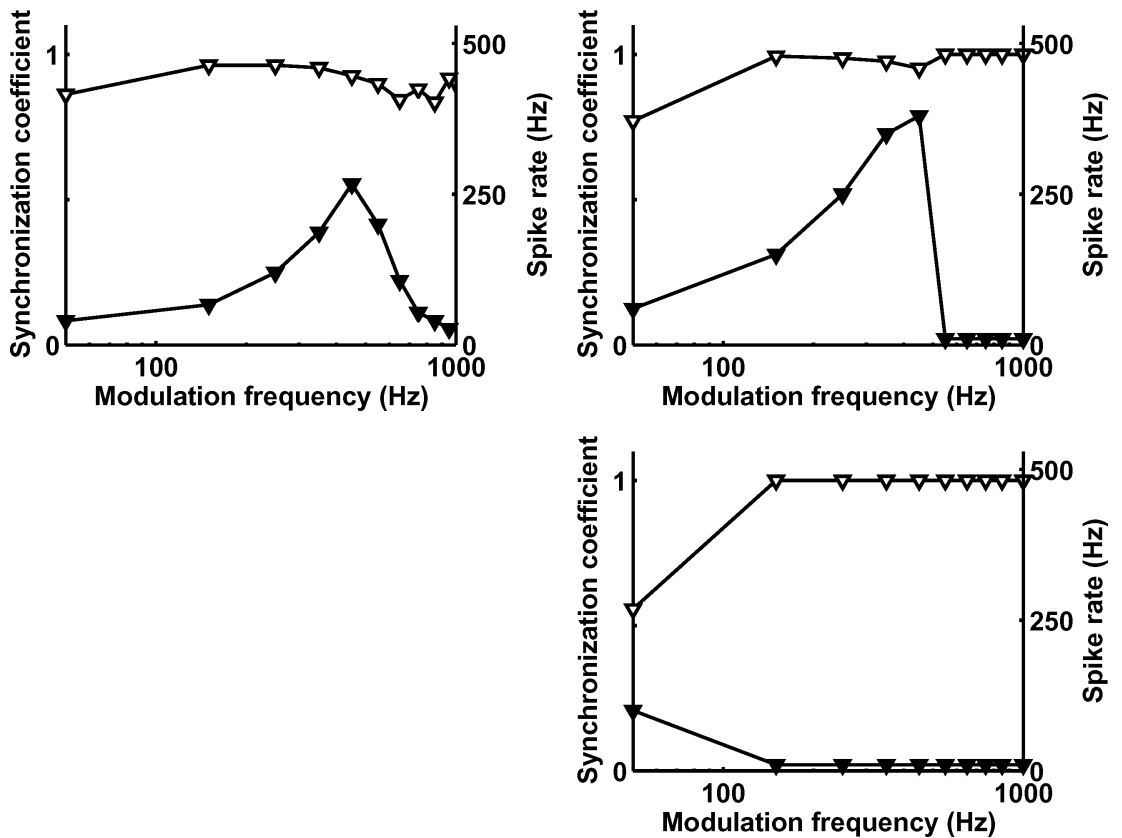

Figure 7. Left panel: Recorded synchronization coefficient (left scale, open triangles) and rate modulation transfer function (right scale, filled triangles) of an $O_{I}$ unit $(\mathrm{CF}=7 \mathrm{kHz})$ in response to $200 \%$ amplitude modulated $7-\mathrm{kHz}$ tones presented at $30 \mathrm{~dB}$ above the unit's pure tone threshold (stimulus duration $100 \mathrm{~ms}$ ). Replot with permission from Rhode (1994). Upper right panel: Simulated synchronization coefficients (left scale, open triangles) and rate modulation transfer functions (right scale, filled triangles) of the present model. The model unit $(\mathrm{CF}=$ $7 \mathrm{kHz}$ ) was tested using 200\% amplitude modulated 7-kHz tones presented at $30 \mathrm{~dB}$ (mTh) (stimulus duration $100 \mathrm{~ms}$ ). Lower right panel: Corresponding simulations obtained with the leaky-integrator model.

in the modulated tones only if the stimulus level is below $10 \mathrm{~dB}$ (mTh) (not shown).

\section{Discussion}

\subsection{Simplifications Made in the Present Model}

The main motivation of the present study was to simulate the spiking behavior of $O_{I}$-units in the PVCN in response to a set of different stimuli. An abstract model with no direct correspondence to biophysical processes was described. Several strongly simplifying assumptions were made within the framework of the model. For example, the simulated membrane potential of the model does not reflect the refractoriness of the unit. Refractoriness is implemented in the model cells using a fixed refractory period and the spike-blocking mechanism. Thus, refractoriness is not reflected by setting the membrane potential to a value below the resting potential of the unit after each spike since such a mechanism would disturb the spike-blocking mecha- nism of the present model. A more realistic shape of the membrane potential might be achieved by assuming two potentials: one that explicitly involves refractoriness and another one that drives the spike-blocking mechanism and is not affected by refractoriness. The implementation of such an extra model potential was not attempted here in order to keep the model simple without affecting its spike generation properties.

A similar spike-blocking mechanism as the one implemented by Kalluri and Delgutte (2003b) was assumed in the present study. It is unclear whether there is a biophysical basis for such a mechanism. It might be associated with an inactivation of $\mathrm{Na}^{+}$channels, as these channels are among the principal channels underlying fast spiking in neurons. The inactivation of $\mathrm{Na}^{+}$channels (depolarization block) was originally suggested by Romand (1978) as a mechanism shaping onset responses. While the spike-blocking mechanism is driven by the magnitude of the stimulus in the model by Kalluri and Delgutte (2003b), it is effectively driven by the stimulus slope in the present model. 
In the peripheral stage of the present model, the mechanical to neural transduction at the hair cell-auditory nerve synapse was simulated using Meddis' model implementation (Meddis, 1986, 1988; Meddis et al., 1990). Instead of actually computing individual spike trains for each AN fiber the "deterministic" rate function at the output of the synapse was considered as representing the AN activity. This only represents a good approximation of the summed activity of a very large number of stochastically firing AN fibers. The actual number of AN fibers projecting onto an octopus cell has been difficult to quantify. While (Liberman, 1993) estimated that approx. 63 AN fibers project onto the soma of an octopus cell, the total number of AN fibers terminating on an octopus cell is presumably several times higher since AN fiber synapses are also located on the dendrites of these cells (Kane, 1973; Smith and Rhode, 1989; Oertel et al., 2000). Based on the fraction of the somatic and dendritic surface area of octopus cells that is covered by synaptic terminals and the average size of synaptic terminals on octopus cells, Kalluri and Delgutte $(2003 \mathrm{a}, \mathrm{b})$ estimated a number of up to 600 AN fiber synapses per octopus cell.

Some of the discrepancies between model predictions and recordings are most likely due to the use of the deterministic nature of the current model implementation. In the present form the model produces essentially no dynamic range and the predicted spike rates in response to high-entrainment frequencies clearly exceed the recorded spike rates in the data (see Fig. 4). If a stochastic component would be included in the model, such as stochastically firing AN fibers or a stochastic spike threshold, the predicted dynamic range of the unit can be expected to be somewhat increased since it would deviate from its all-or-nothing behavior at stimulus levels near threshold. In addition, a stochastic element would limit entrainment and would decrease the spike rate at high entrainment frequencies (see Fig. 4) where the fluctuations in the membrane potential are already very weak. These aspects need to be examined explicitly in further modelling efforts. However, the present study was focussed on investigating the fundamental differences between a leaky-integration and a change-detection approach whereby the same (deterministic) AN input was used in both model implementations. The main differences between these two models will persist even when individual AN spike trains are used instead of the discharge rate.

The actual frequency range of AN fibers that provide input to one octopus cell is not known yet. However, estimates in mice suggest a frequency range of about 2-3 octaves (Oertel et al., 2000). Compared to this estimate, the input frequency range assumed in the present model unit (about one octave) is rather small. An extension of the across-frequency range of the model input, especially for units with a low CF is restricted by the different traveling-wave delays on the basilar membrane. Large differences in the traveling-wave delays between channels, that provide input to the same octopus cell, would distort the input shape due to destructive interference. This might lead to inappropriate model responses. However, octopus cells were found to receive input from $\mathrm{AN}$ fibers that encode low frequencies near their cell body and input from fibers that encode higher frequencies progressively more distally on their dendrites (Oertel et al., 2000). This ordered spatial arrangement has been suggested to compensate for the traveling-wave "distortion" along the cochlea and to increase the synchronization of across-frequency input (Golding et al., 1999). Extending the present model by such a travelling-wave compensation may provide a simple way of extending the across-frequency range of AN inputs.

It is not possible to directly correlate the shape of the assumed impulse response in the model with distinct ion conductances. However, the biphasic shape that reflects an intrinsic model property might result from combined fast inwardly directed currents and slower outwardly directed currents, in response to changes of the membrane potential. Recent studies focusing on the intracellular properties of octopus cells reveal that an outward rectifying low-threshold, depolarizationactivated potassium conductance plays a critical role in shaping octopus cells responses (Golding et al., 1999). Whether the negative part of the biphasic impulse response reflects the effective contribution of this outwardly directed low-threshold potassium conductance cannot be decided here.

\subsection{Change Detection Versus Stimulus Integration}

Octopus cells have been assumed to act as coincidence detectors (e.g., Oertel et al., 2000) due to the large number of high-spontaneous rate AN fibers (Liberman, 1993) providing across-frequency excitatory AN input and due to their rapid membrane time constant (<200 $\mu \mathrm{s}$ ) (Golding et al. 1995, 1999). However, as was already shown by Kalluri and Delgutte (2003a), coincidence detection itself is not sufficient to simulate the ideal onset responses to pure tones observed in 
octopus cells. Moreover, the results of the present study show that the leaky-integrator dynamic underlying the coincidence-detector model by Kalluri and Delgutte (2003a) differs fundamentally from recorded octopus cell responses. While octopus cells have been found to detect the rate of change in injected currents (Ferragamo and Oertel, 2002), the leaky-integrator model evaluated here is not affected by the rate of change in the current stimulus (see Fig. 5). In addition, neither the hyperpolarization observed in an octopus cell's potential after stimulus offset (e.g., Golding et al., 1999) nor the offset potential observed in response to hyperpolarizing current steps (Oertel et al., 2000) can be described by this model. When combined with a spikeblocking mechanism, the leaky-integrator model was shown to be able to account for some of the responses of ideal onset units to pure tones (Kalluri and Delgutte, 2003b). However, the model is still unable to account for octopus cell responses to increasing current steps (Fig. 6). While an octopus cell generates an action potential with each current increment (Oertel et al., 2000), the leaky-integrator model combined with the spikeblocking mechanism generates only an action potential at the onset of the staircase current. The simulated frequency-response area of the leaky-integrator model (Fig. 4) indicates another problem, resulting from the fixed spike-blocking release threshold in the model: at a given stimulus level, there is only a small range of frequencies where the fluctuations in the membrane potential exceed the potential difference between the spike threshold and the spike-blocking release threshold; i.e., entrainment is restricted to this small frequency range. Moreover, in contrast to recorded octopus cell responses, there is a trade-off between the effects of stimulus level and the highest frequency to which the model unit entrains. A similar problem occurs when the leaky-integrator model is tested using $200 \%$ amplitude modulated pure-tones. The model fails to detect the fluctuations in the stimulus envelope and exhibits only an onset response, independent of the modulation frequency (Fig. 7).

Assuming a biphasic membrane impulse response (in combination with a spike-blocking mechanism), octopus cell responses can be described, at least qualitatively, in the different stimulus conditions. The predicted PSTHs show a single spike at the onset of a highfrequency tone burst and no further activity during the ongoing stimulation. The same simulated units show entrainment to a wide range of low-frequency tones, revealing exactly one spike per stimulus cycle and thus exhibiting inter-spike intervals that are in the range of milliseconds. The model also accounts for the main characteristics in the responses to the injected currents and to the amplitude modulated tones. This results from the higher sensitivity of this model to stimulus transients and fast changes relative to sustained stimulus portions.

The model in the present form, however, does not account for integration of depolarization, e.g., when stimulated with current steps of increasing strength (Oertel et al., 2000). A pure change detector was used in the present investigation, i.e., the constant $c$ in Eq. (2) was chosen such that the integral of the biphasic impulse response over time is zero. Stimulus integration can, however, easily be included by decreasing the value of $c$. Figure 8 shows simulation results obtained with a value of $c=0.232$. Within the simulations the spike-blocking release potential was changed to $\Theta_{\text {rel }}=-48 \mathrm{mV}$, while all other parameters of the model remained unchanged. The upper left panel (Fig. 8A) shows the corresponding impulse response (solid line) together with a replot of the original impulse response (dashed line). The two corresponding transfer functions are shown in Fig. 8B. The simulations obtained with the modified parameter for the staircase current are shown in Fig. 8C. While the spikingbehavior of the model remains unchanged, the membrane potential in the plateau regions of the current steps corresponds to the recorded octopus cell potential (compare left panel of Fig 6). Figure 8D shows the simulated response to currents of different strengths and rise times (comp. Fig. 5). Although the model response is not as distinct as for the original simulation without integration property (the $2.5 \mathrm{nA}$ current rising over $1.2 \mathrm{~ms}$ nearly brings the unit to threshold), the model still reveals the same spiking behavior as seen in Fig. 5. Figure 8E and F show the simulated response to the same pure tones as used in Fig. 3. Although the impulse response of the model includes some stimulus integration, it still reveals the same spiking behavior (comp. Fig. 3). This is also true for the simulated frequency-response area and the rMTFs (not shown) that reveal no major differences compared to the simulations obtained with the original model. However, the model's entrainment to medium frequencies and, thus, its peak spike-rate, are increased since stimulus integration leads to an increased depolarization of the model's membrane. Such an enhanced peak spike-rate could probably be avoided by changing the spike threshold within the model, if stimulus integration is desired to 

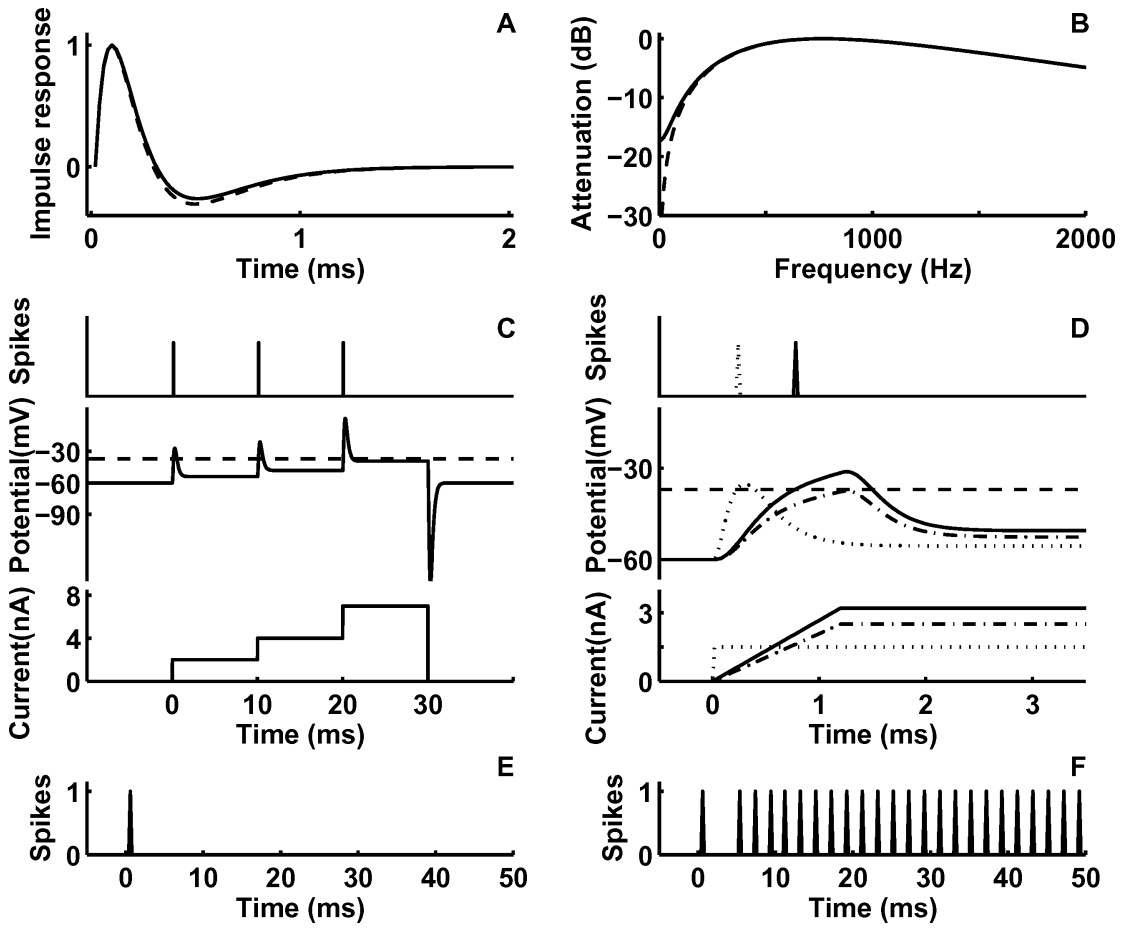

Figure 8. Simulated responses of a model assuming a biphasic impulse response that includes stimulus integration $(c=0.232$ in Eq. $(2)$ and $\Theta_{\text {rel }}=-48 \mathrm{mV}$ ). All other model parameters remained unchanged. Panel A: Impulse response including stimulus integration (solid line) and original impulse response (dashed line). Panel B: Corresponding frequency transfer functions. Panel C: Simulation results in response to a staircase current as used in Fig. 6. Panel D: Simulation results in response to currents of different strengths and rise times as used in Fig. 5. Panel E. Simulated PSTH in response to a 4-kHz tone as used in Fig. 3. Panel F. Simulated PSTH in response to a 500-Hz tone.

be included. The additional simulations indicate that stimulus integration can, in principle, easily be introduced without changing the spiking behavior. As long as only the spiking behavior is considered, the pure change detector seems sufficient for the simulation of the octopus cell responses in the experimental conditions investigated here.

In summary, a functional point-neuron model was presented that describes $O_{I}$-unit responses found in cochlear nucleus octopus cells. The model correctly describes some of the characteristics of the spiking behavior of these neurons in response to pure tones at low and high frequencies, injected currents, and amplitudemodulated tones, using the same small set of parameters for all experimental conditions. The data cannot be accounted for by a leaky-integrator that acts as a coincidence detector. The present model is similar to previous models (e.g., Cai et al., 1997; Levy and Kipke, 1997; Kalluri and Delgutte, 2003b) in terms of its short time constants (below $1 \mathrm{~ms}$ ). However, within the present model, these short time constants are not associated with a coincidence-detection mechanism, but instead are required to allow for an entrainment to high input frequencies. Overall, the results from the present study indicate that some of the response properties observed in $O_{I}$-units can be understood from relatively simple principles. The present model might be useful as a processing module in more complex models of higher-order auditory processing and perception.

\section{Acknowledgments}

We thank two anonymous reviewers for their very helpful remarks and suggestions. We would like to thank Birger Kollmeier and his group "Medizinische Physik," Stephan Ewert, and Paul Nelson for fruitful discussions about the content of this paper. This work was supported by the Deutsche Forschungsgemeinschaft (DFG), Research project GRK 591 ("International Graduate School for Neurosensory Science and Systems") and Research project SPP 1046 ("Temporal processing in the central auditory system"). 


\section{References}

Bal R, Oertel D (2001) Potassium currents in octopus cells of the mammalian cochlear nucleus. J. Neurophysiol. 86: 2299-2311.

Bregman AS (1990) Auditory Scene Analysis: The Perceptual Organization of Sound. MIT Press, Cambridge.

Cai Y, Walsh EJ, McGee J (1997) Mechanisms of onset responses in octopus cells of the cochlear nucleus: Implications of a model. J. Neurophysiol. 78: 872-883.

Cai Y, McGee J, Walsh EJ (2000) Contributions of ion conductances to the onset responses of octopus cells in the ventral cochlear nucleus: Simulation results. J. Neurophysiol. 83: 301-314.

Cai Y, McGee J, Walsh EJ (2001) Processing of pitch information in complex stimuli by a model of octopus cells in the cochlear nucleus. In: S Greenberg, M Slaney, eds., Computational Models of Auditory Function. IOS Press, Amsterdam.

Ferragamo MJ, Oertel, D (2002) Octopus cells of the mammalian ventral cochlear nucleus sense the rate of depolarization. J. Neurophysiol. 87: 2262-2270.

Frisina RD, Smith RL, Chamberlain SC (1990) Encoding of amplitude modulation in the gerbil cochlear nucleus: I. A hierarchy of enhancement. Hear. Res. 44: 99-122.

Gardner SM, Trussell LO, Oertel D (1999) Time course and permeation of synaptic AMPA receptors in cochlear nucleus neurons correlate with input. J. Neurosci. 19: 8721-8729.

Godfrey DA, Kiang NYS, Norris BE (1975) Single unit activity in the posteroventral cochlear nucleus of the cat. J. Comp. Neurol. 162: $247-268$

Goldberg JM, Brown PB (1969) Responses of binaural neurons of dog superior olivary complex to dichotic stimuli: Some physiological mechanisms of sound localization. J. Neurophysiol. 32: 613-636.

Golding NL, Robertson D, Oertel D (1995) Recordings from slices indicate that octopus cells of the cochlear nucleus detect coincident firing of auditory nerve fibers with temporal precision. J. Neurosci. 15: $3138-3153$

Golding NL, Ferragamo MJ, Oertel D (1999) Role of intrinsic conductances underlying responses to transients in octopus cells of the cochlear nucleus. J. Neurosci. 19: 2897-2905.

Kalluri S, Delgutte B (2003a) Mathematical models of cochlear nucleus onset neurons: I. Point neuron with many weak synaptic inputs. J. Comp. Neurosci. 14: 71-90.

Kalluri S, Delgutte B (2003b) Mathematical models of cochlear nucleus onset neurons: II. Model with dynamic spike-blocking state. J. Comp. Neurosci. 14: 91-110.

Kane EC (1973) Octopus cells in the cochlear nuclei of the cat: Heterotypic synapses upon homeotypic neurons. Int. J. Neurosci. 5: 251-279.
Kipke DR, Levy KL (1997) Sensitivity of the cochlear nucleus octopus cell to synaptic and membrane properties. J. Acoust. Soc. Am. 102: 403-412.

Levy KL, Kipke DR (1997) A computational model of the cochlear nucleus octopus cell. J. Acoust. Soc. Am. 102: 391-402.

Levy KL, Kipke DR (1998) Mechanisms of the cochlear nucleus octopus cell's onset response: Synaptic effectiveness and threshold. J. Accoust. Soc. Am. 103: 1940-1950.

Liberman MC (1993) Central projections of auditory nerve fibers of differing spontaneous rate. II: Posteroventral and dorsal cochlear nuclei. J. Comp. Neurol. 327: 17-36.

Meddis R, Hewitt MJ, Shackleton TM (1990) Implementation details of a computation model of the inner hair-cell/auditory-nerve synapse. J. Acoust. Soc. Am. 87: 1813-1816.

Meddis R (1986) Simulation of mechanical to neural transduction in the auditory receptor. J. Acoust. Soc. Am. 79: 702-711.

Meddis R (1988) Simulation of auditory-neural transduction: Further studies. J. Acoust. Soc. Am. 83: 1056-1063.

Oertel D, Bal R, Gardner SM, Smith PH, Joris PX (2000) Detection of synchrony in the activity of auditory nerve fibers by octopus cells of the mammalian cochlear nucleus. PNAS. National Academy of Sciences Colloquium on Auditory Neuroscience: Development, Transduction and Integration .

Patterson RD, Nimmo-Smith I, Holdsworth J, Rice P (1988) An efficient auditory filterbank based on the gammatone function. APU report 2341, Applied Psychology Unit, Cambridge.

Rhode WS, Smith PH (1986) Encoding timing and intensity in the ventral cochlear nucleus of the cat. J. Neurophysiol. 56: 261286.

Rhode WS (1994) Temporal coding of $200 \%$ amplitude modulated signals in the ventral cochlear nucleus of cat. Hear. Res. 77: 43-68.

Romand R (1978) Survey of intracellular recording in the cochlear nucleus of the cat. Brain Res. 148: 43-65.

Smith PH, Rhode WS (1989) Structural and functional properties distinguish two types of multipolar cells in the ventral cochlear nucleus. J. Comp. Neurol. 282: 595-616.

Stevens KN (1995) Applying phonetic knowledge to lexical access. In: Fourth European Conference on Speech Communication and Technology, Vol. 1. Madrid, Spain.

Tuckwell HC (1988) Introduction to Theoretical Neurobiology, Vol. 1. Cambridge University Press, Cambridge, England.

Winter IM, Palmer AR (1995) Level dependence of cochlear nucleus onset unit responses and facilitation by second tones or broadband noise. J. Neurophysiol. 73: 141-159.

Young E, Robert JM, Shofner W (1988) Regularity and latency of units in ventral cochlear nucleus: Implications for unit classification and generation of response properties. J. Neurophysiol. 60: $1-29$. 